\title{
Gastric IgG4-related disease presenting as a mass lesion and masquerading as a gastrointestinal stromal tumor
}

\author{
Banumathi Ramakrishna ${ }^{1}$, Rohan Yewale ${ }^{2}$, Kavita Vijayakumar ${ }^{1}$, Patta Radhakrishna ${ }^{3}$, Balakrishnan Siddartha Ramakrishna ${ }^{2}$ \\ Departments of ${ }^{1}$ Pathology, ${ }^{2}$ Medical Gastroenterology, and ${ }^{3}$ Surgical Gastroenterology, SRM Institutes for Medical Science, Vadapalani, India
}

\begin{abstract}
lgG4-related disease of the stomach is a rare disorder, and only a few cases have been reported. We present two cases that were identified over a 2-month period in our center. Two male patients aged 52 and 48 years presented with mass lesion in the stomach, which were clinically thought to be gastrointestinal stromal tumor, and they underwent excision of the lesion. Microscopic examination revealed marked fibrosis, which was storiform in one case, associated with diffuse lymphoplasmacytic infiltration and an increase in lgG4positive plasma cells on immunohistochemistry. Serum lgG4 level was markedly elevated. Although rare, IgG4-related disease should be considered in the differential diagnosis of gastric submucosal mass lesions.
\end{abstract}

Key Words: IgG4-related disease; Stomach; Autoimmune diseases; Gastrointestinal stromal tumors

Received: December 4, 2019 Revised: February 5, 2020 Accepted: February 10, 2020

Corresponding Author: Banumathi Ramakrishna, MBBS, MD, Department of Pathology, SRM Institutes for Medical Science, 1 Jawaharlal Nehru Road, Vadapalani, Chennai 600026, India

Tel: +91-44-20002001, Fax: +91-44-49211455, E-mail: banu_ramakrishna@hotmail.com

IgG4-related disease (IgG4-RD) is a newly-recognized, immune-mediated fibroinflammatory disorder that most commonly affects the pancreas [1]. It can also involve other organs such as the bile duct, liver, gallbladder, salivary glands, lacrimal glands, retroperitoneum, and lymph nodes, although involvement of the gastrointestinal tract is very rare $[2,3]$. The disorder can present as diffuse wall thickening or as polyp or mass-like lesion [2]. Histologically, this disease is characterized by storiform fibrosis, diffuse lymphoplasmacytic infiltrates, obliterative or non-obliterative phlebitis, increase in IgG4-positive plasma cells on immunostaining, and often with elevated serum IgG4 level $[1,3,4]$. We report two cases of IgG4-RD of the stomach that presented as a mass lesion, were identified over a two-month period, and were clinically suspected to be gastrointestinal stromal tumor (GIST) and surgically excised.

\section{CASE REPORT}

\section{Case 1}

A 42-year-old male patient was admitted to our hospital with a diagnosis of acute pyelonephritis. Computed tomography
(CT) of the kidney, ureter, and bladder revealed an incidental mass lesion in the posterior wall of the stomach. Upper gastrointestinal endoscopy revealed submucosal lesions in the esophagus and stomach that were clinically suspected to be GIST. The patient underwent endoscopic ultrasound-guided fine-needle aspiration biopsy of the esophageal lesion and endoscopic mucosal biopsy of the stomach, both of which were inconclusive. The patient also underwent wedge resection of the gastric lesion. Histopathological examination revealed a wedge of the gastric wall with a globular submucosal lesion measuring $6.5 \times 6.0 \times 4.0 \mathrm{~cm}$. The cut surface had a greyish white appearance with foci of calcification. Microscopically, there was marked fibrous expansion of the submucosa with collagenization extending through the muscularis propria to the subserosa, diffuse infiltrates of predominantly plasma cells arranged in cords and clusters admixed with lymphocytes and eosinophils, and several lymphoid follicles with reactive germinal centers (Fig. 1A, B). Small foci of calcification were also present. The muscle coat was disorganized and had muscular hypertrophy in foci. There was no storiform fibrosis or evidence of obliterative or non-obliterative phlebitis. Based on these characteristics, a possible diagnosis of IgG4-RD was suggested. 

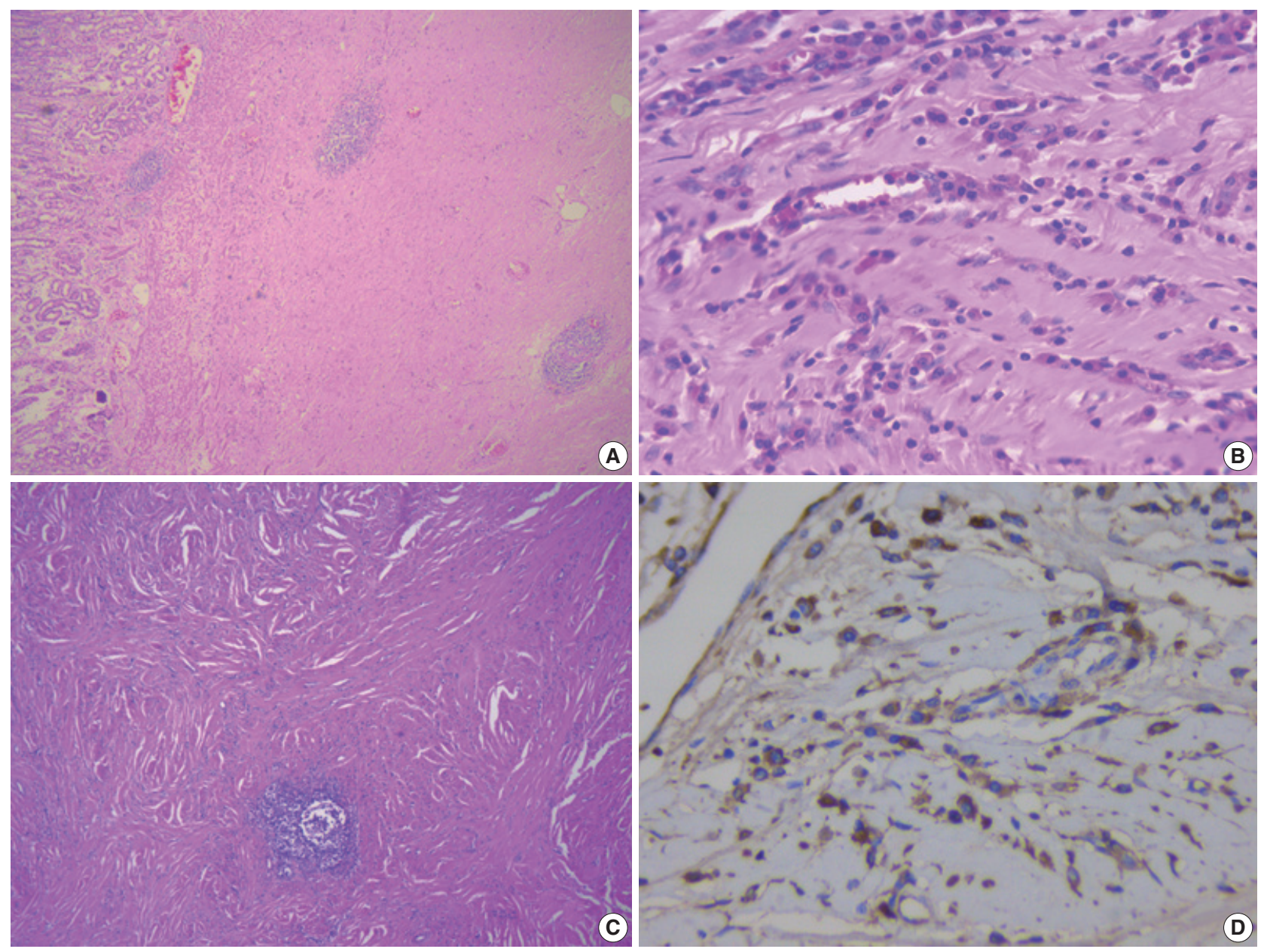

Fig. 1. (A) Gastric wall with marked submucosal fibrosis and prominent lymphoid follicles. (B) Diffuse plasma cell rich inflammation. (C) Storiform fibrosis with diffuse inflammation and a lymphoid follicle. (D) Many immunoreactive lgG4-positive plasma cells.

IgG4 immunohistochemistry (IHC) and serum IgG4 level were assessed. The IgG4 IHC showed 35-40 immunoreactive plasma cells per high power field (hpf), and serum IgG4 level was elevated $(4.36 \mathrm{~g} / \mathrm{L})$. These tests confirmed the diagnosis of IgG4-RD. Subsequent serum levels after 3 and 4 months were $2.71 \mathrm{~g} / \mathrm{L}$ and $2.53 \mathrm{~g} / \mathrm{L}$, respectively. The patient was treated with steroids and azathioprine. He experienced postsurgery complications that required revision of the gastric anastomosis. During follow-up, his prognosis while receiving medical treatment has been good.

\section{Case 2}

The second patient was a 58-year-old male found to have erosive gastritis and a submucosal swelling in the body of the stomach in December 2017, while undergoing upper gastrointestinal endoscopy for investigation of dyspepsia. CT examination showed a well-defined, $3 \times 2.9 \mathrm{~cm}$, round, homogeneous, en- hancing soft tissue lesion in the distal body of the stomach along the lesser curvature, which was suspected to be GIST or leiomyoma. He was advised to undergo follow-up and elective surgery. His gastric symptoms worsened over the next 10 months, and he underwent excision of the gastric submucosal lesion in October 2018. Histopathological examination revealed a wellcircumscribed globular mass measuring $4 \times 3.3 \times 3.5 \mathrm{~cm}$, and whorling was seen on the cut surface. Microscopically, the lesion was composed of extensively fibrotic and sclerotic stroma with a storiform pattern of fibrosis in foci (Fig. 1C). Discrete, cords and clusters of plasma cells admixed with lymphocytes, a few eosinophils and a few scattered lymphoid aggregates and follicles were present. Perivascular aggregates of plasma cells were also present. There was no evidence of obliterative or nonobliterative phlebitis. Bundles of smooth muscle were identified at the periphery on one aspect. The possibility of IgG4-RD of the stomach was suggested, and $\mathrm{IgG} 4 \mathrm{IHC}$ and serum estima- 
tion were recommended. The IgG4 IHC revealed 20-30 immuno-reactive plasma cells/hpf (Fig. 1D). Serum IgG4 was elevated at $3.11 \mathrm{~g} / \mathrm{L}$, well above the $1.35 \mathrm{~g} / \mathrm{L}$ cut-off for diagnosis of IgG4-RD. The patient did not return for follow-up.

\section{Ethics statement}

The authors certify that they obtained patient consent for publication, and the study was approved by the Institutional Review Board of Sri Ramaswamy Memorial Institutes for Medical Science, Chennai, India (IEC NO: SIMS IEC/other/18/2019).

\section{DISCUSSION}

IgG4-RD is an immune-mediated fibroinflammatory lesion, first described in patients with sclerosing cholangitis associated with autoimmune pancreatitis type 1 [5]. Later, it was identified in other organs including the liver, bile ducts, salivary glands, retroperitoneum, lymph nodes, and lungs. It is characterized by diffuse or partial enlargement of the organ and histologically as dense lymphoplasmacytic infiltration with an increase in IgG4 plasma cells on immunostaining, a storiform pattern of fibrosis and obliterative or non-obliterative phlebitis, and an increase in serum IgG4 level.

IgG4-RD of the gastrointestinal tract is very rare and can present as diffuse wall thickening or as polyp or mass-like lesion $[2,3]$. Even though obliterative phlebitis was not present in these two cases, the presence of dense fibrosis, which was storiform in one case, and dense lymphoplasmacytic infiltration with lymphoid aggregates and follicles, presenting as a submucosal masslike lesion, suggested the possibility of IgG4-RD, which was confirmed by IHC and elevated serum IgG4 level. The presence of at least two histological features is required for confident diagnosis of IgG4-RD; in most cases, dense lymphoplasmacytic infiltrate and diffuse/storiform fibrosis are seen. Additional clinical, serological (serum level $>135 \mathrm{mg} / \mathrm{dL}$ or $1.35 \mathrm{~g} / \mathrm{L}$ ), or radiological evidence is required to confirm IgG4-RD [1].

Table 1. Clinicopathological features of lgG4-related disease manifesting as gastric lesions

\begin{tabular}{|c|c|c|c|c|c|c|c|c|c|}
\hline Case No. & $\begin{array}{l}\text { Age } \\
\text { (yr) }\end{array}$ & Sex & $\begin{array}{l}\text { Endoscopic finding/ } \\
\text { Clinical diagnosis }\end{array}$ & Location & $\begin{array}{l}\text { Size } \\
(\mathrm{mm})\end{array}$ & Histopathology/lHC & $\begin{array}{l}\text { Serum } \\
\text { lgG4 levels }\end{array}$ & Treatment & Study \\
\hline 1 & 48 & $F$ & Mass/GIST/NET & Mid body & $36 \times 22$ & $\begin{array}{l}\text { SF, LP, OP, lgG4 +210/hpf, } \\
\text { lgG4/lgG ratio about } 85 \%\end{array}$ & $\mathrm{NA}$ & WR & Woo et al. [3] \\
\hline 2 & 62 & F & Mass/gastic cancer & Antrum & $80 \times 30$ & $\begin{array}{l}\text { SF, LP, OP, IgG4 + ve } \\
\text { lymphoplasmacytes > 50/hpf }\end{array}$ & Elevated & $D G$ & Bulanov et al. [6] \\
\hline 3 & 59 & $\mathrm{~F}$ & Mass/GIST & NA & $33 \times 14$ & $\begin{array}{l}\text { Abundant LP, SF, lymphoid } \\
\text { follicles, lgG4 > 50/hpf }\end{array}$ & Normal & WR & Kim et al. [7] \\
\hline 4 & 56 & $\mathrm{~F}$ & Mass/GIST & NA & $21 \times 15$ & $\begin{array}{l}\text { Abundant LP, SF, calcification, } \\
\operatorname{lgG} 4>50 / \mathrm{hpf}\end{array}$ & Normal & WR & Kim et al. [7] \\
\hline 5 & 60 & F & Nodule/NA & Fundus & $10 \times 15$ & $\begin{array}{l}\text { Fibrosis, dense LP, } \\
\operatorname{lgG} 4>80 / \mathrm{hpf}\end{array}$ & Normal & WR & Chetty et al. [8] \\
\hline 6 & 45 & M & Multiple nodules/NA & Antrum & Up to 22 & $\begin{array}{l}\text { LP, many eosinophils, } \\
\text { lgG4/lgG ratio } 0.84\end{array}$ & NA & $D G$ & Chetty et al. [8] \\
\hline 7 & 56 & M & Nodule/NA & Body & 8 & $\begin{array}{l}\text { SF, LP, IgG4-40-102/hpf } \\
\text { IgG4/lgG ratio 80\%-90\% }\end{array}$ & NA & ESR & Na et al. [9] \\
\hline 8 & 58 & M & Nodule/AIP & Fundus and body & 14 & $\begin{array}{l}\text { Dense LP, extensive } \lg G \text { and } \\
\lg G 4 \text { + staining }\end{array}$ & Normal & Steroid & Baez et al. [10] \\
\hline 9 & 55 & $F$ & Nodule/GIST & Body & 20 & $\begin{array}{l}\text { Dense hyalinization, LP, } \\
\text { lgG4/lgG ratio } 41 \%\end{array}$ & Normal & ESR & Zhang et al. [11] \\
\hline 10 & 75 & $\mathrm{~F}$ & Polyp/GIST & Body & $56 \times 50$ & $\begin{array}{l}\text { Fibrosis, LP, many eosinophils, } \\
\text { lgG4-39/hpf }\end{array}$ & Normal & WR & Rollins et al. [12] \\
\hline 11 & 44 & M & Mass/GIST & Body & $20 \times 18$ & $\begin{array}{l}\text { Fibrosis, LP, IgG4 + ve } \\
\text { lymphoplasmacytes }\end{array}$ & Normal & ESR & Otsuka et al. [13] \\
\hline 12 & 27 & $\mathrm{~F}$ & Mass/GIST/NET & Fundus & 40 & $\begin{array}{l}\text { Dense fibrosis, LP, lgG4/lgG } \\
\text { ratio } 25.3 \%\end{array}$ & Normal & WR & Cheong et al. [14] \\
\hline 13 & 29 & $\mathrm{~F}$ & Mass/GIST & Body & $20 \times 15$ & $\begin{array}{l}\text { Fibrosis, LP, lgG4 + ve plasma } \\
\text { cells } 150 / \mathrm{hpf}\end{array}$ & NA & WR & Skorus et al. [15] \\
\hline 14 & 43 & M & Mass/GIST & Antrum & $70 \times 50$ & $\begin{array}{l}\text { Dense LP, lgG4 + plasma cells } \\
\text { 35-40/hpf }\end{array}$ & Elevated & $\begin{array}{l}\text { WR }+ \\
\text { Steroids }\end{array}$ & Present case 1 \\
\hline 15 & 58 & M & Mass/GIST & Distal body & $45 \times 40$ & $\begin{array}{l}\text { LP, SP, lgG4 + ve plasma cells } \\
20-30 / h p f\end{array}$ & Elevated & WR & Present case 2 \\
\hline
\end{tabular}

IHC, immunohistochemistry; GIST, gastrointestinal stromal tumor; NET, neuroendocrine tumor; SF, storiform fibrosis; LP, lymphoplasmacytic infiltrate; OP, obliterative phlebitis; hpf, high power field; NA, not available; WR, wedge resection; DG, distal gastrectomy; ESR, endoscopic submucosal resection; AIP, autoimmune pancreatitis. 
The cut-off point for the presence of IgG4 plasma cells in tissues varies and can range from $>30$ plasma cells/hpf to $>50 / \mathrm{hpf}$, which is highly specific $[1,5]$. In biopsy specimens, more than 10 IgG4 plasma cells/hpf were reported in one study [16]. However, the cut-off points vary depending on organ system. Some studies have suggested that $\operatorname{IgG} 4^{+} / \mathrm{IgG}^{+}$plasma cell ratio $>0.4$ is a marker of IgG4-RD in the presence of classic histopathological features and with a compatible clinical features [1,17].

IgG4-RD can involve multiple organs or any sites in the body synchronously or metachronously [18]. Patients can present with non-specific symptoms and swelling or mass-like lesion. Patients with biliary or pancreatic lesion can present with jaundice, weight loss, and vague abdominal pain. The disease can be an incidental finding during radiological examination and can be mistaken for malignancy, as there are no specific radiological features characteristic of this disease $[18,19]$. Most cases of gastric IgG4-RD have been reported in middle-aged patients, and both men and women are affected $[3,6]$. Both patients in this report were middle-aged men.

IgG4-RD of the stomach was first described in 2004 by Shinji et al. [20], presenting as a gastric ulcer. Because it is difficult to diagnose clinically, especially in isolated cases, most of the reported patients have undergone surgery. Because this disease involves a submucosal lesion in the stomach, these cases are often misdiagnosed as GIST $[3,7,18,19]$ and are difficult to diagnose on endoscopic forceps biopsy, similar to our cases. Gastric lesions that have been mistaken for GIST or gastric cancer have been reported in the literature (Table 1) [3,6-15]. IgG4-RD of the stomach that involved the regional lymph nodes has also been reported [6].

Although steroids are the first therapeutic option for treating IgG4-RD, it is difficult to diagnose gastric IgG4-RD without histopathological examination. Almost all cases have been reported after surgical resection. Therefore, this disease should be considered in the differential diagnosis of gastric submucosal mass lesion.

To conclude, we present two cases of IgG4-RD of the stomach that presented as a mass lesion and were clinically suspected to be GIST. The diagnosis was made only after histopathological examination of resection specimens. This highlights the importance of considering this disease in differential diagnosis to avoid surgical resection.

\section{ORCID}

Banumathi Ramakrishna:

https://orcid.org/0000-0001-5635-1633
Balakrishnan Siddartha Ramakrishna:

https://orcid.org/0000-0001-5090-9501

\section{Author Contributions}

Conceptualization: BR.

Data curation: BR, RY.

Investigation: BR, KV, PR, RY, BSR.

Visualization: BR.

Writing—original draft: BR

Writing—review and editing: BR, RY, BSR.

\section{Conflicts of Interest}

The authors declare that they have no potential conflicts of interest.

\section{Funding}

No funding to declare.

\section{REFERENCES}

1. Deshpande V, Zen Y, Chan JK, et al. Consensus statement on the pathology of IgG4-related disease. Mod Pathol 2012; 25: 1181-92.

2. Koizumi S, Kamisawa T, Kuruma S, et al. Immunoglobulin G4-related gastrointestinal diseases, are they immunoglobulin G4-related diseases? World J Gastroenterol 2013; 19: 5769-74.

3. Woo CG, Yook JH, Kim AY, Kim J. IgG4-related disease presented as a mural mass in the stomach. J Pathol Transl Med 2016; 50: 67-70.

4. Stone JH, Zen Y, Deshpande V. IgG4-related disease. N Engl J Med 2012; 366: 539-51.

5. Kamisawa T, Funata N, Hayashi Y, et al. A new clinicopathological entity of IgG4-related autoimmune disease. J Gastroenterol 2003; 38: 982-4.

6. Bulanov D, Arabadzhieva E, Bonev S, et al. A rare case of IgG4-related disease: a gastric mass, associated with regional lymphadenopathy. BMC Surg 2016; 16: 37.

7. Kim DH, Kim J, Park DH, et al. Immunoglobulin G4-related inflammatory pseudotumor of the stomach. Gastrointest Endosc 2012; 76: 451-2.

8. Chetty R, Serra S, Gauchotte G, Markl B, Agaimy A. Sclerosing nodular lesions of the gastrointestinal tract containing large numbers of IgG4 plasma cells. Pathology 2011; 43: 31-5.

9. Na KY, Sung JY, Jang JY, et al. Gastric nodular lesion caused by IgG4-related disease. Pathol Int 2012; 62: 716-8.

10. Baez JC, Hamilton MJ, Bellizzi A, Mortelé KJ. Gastric involvement in autoimmune pancreatitis: MDCT and histopathologic features. JOP 2010; 11: 610-3. 
11. Zhang H, Jin Z, Ding S. Gastric calcifying fibrous tumor: a case of suspected immunoglobulin G4-related gastric disease. Saudi J Gastroenterol 2015; 21: 423-6.

12. Rollins KE, Mehta SP, O'Donovan M, Safranek PM. Gastric IgG4related autoimmune fibrosclerosing pseudotumour: a novel location. ISRN Gastroenterol 2011; 2011: 873087.

13. Otsuka R, Kano M, Hayashi H, et al. Probable IgG4-related sclerosing disease presenting as a gastric submucosal tumor with an intense tracer uptake on PET/CT: a case report. Surg Case Rep 2016; 2: 33.

14. Cheong HR, Lee BE, Song GA, Kim GH, An SG, Lim W. Immunoglobulin G4-related inflammatory pseudotumor presenting as a solitary mass in the stomach. Clin Endosc 2016; 49: 197-201.

15. Skorus U, Kenig J, Mastalerz K. IgG4-related disease manifesting as an isolated gastric lesion- a literature review. Pol Przegl Chir 2018; 90: 41-5.

16. Chari ST. Diagnosis of autoimmune pancreatitis using its five cardinal features: introducing the Mayo Clinic's HISORt criteria. J Gas- troenterol 2007; 42 Suppl 18: 39-41.

17. Miyabe K, Zen Y, Cornell LD, et al. Gastrointestinal and extra-intestinal manifestations of IgG4-related disease. Gastroenterology 2018; 155: 990-1003.

18. Seo HS, Jung YJ, Park CH, Song KY, Jung ES. IgG4-related disease in the stomach which was confused with gastrointestinal stromal tumor (GIST): two case reports and review of the literature. J Gastric Cancer 2018; 18: 99-107.

19. Inoue D, Yoneda N, Yoshida K, et al. Imaging and pathological features of gastric lesion of immunoglobulin G4-related disease: A case report and review of the recent literature. Mod Rheumatol 2019; 29: 377-82.

20. Shinji A, Sano K, Hamano H, et al. Autoimmune pancreatitis is closely associated with gastric ulcer presenting with abundant IgG4-bearing plasma cell infiltration. Gastrointest Endosc 2004; 59: 506-11. 